\title{
¿Cómo generalizar los ciclos de mejora en aula en la asignatura de Anatomía Patológica integrando la docencia teórica y práctica?
}

\section{How to generalize classroom- based learning improvement cycles in the subject of Pathology by integrating the theoretical and practical teaching?}

EnRIQUe de Álava CASAdo

ORCID: https://orcid.org/0000-0001-8400-046X

Universidad de Sevilla

Departamento de Citología e Histología Normal y Patológica y UGC Anatomía Patológica

Hospital Universitario Virgen del

Rocio-IBiS / AGS Osuna

Correo electrónico: ealava-ibis@us.es

Fecha de recepción: 24-11-2019

Fecha de aceptación: 26-11-2019

DOI: http://dx.doi.org/10.12795/9788447221912.076

Pp.: 1753-1776 


\section{Resumen}

Nuestra hipótesis es que el aprendizaje de los alumnos mejoraráía si: i) se integrasen mejor los contenidos procedimentales con los conceptuales a lo largo de ciclos de mejora en aula (CIMA) que rompan la dicotomía tradicional entre clases teóricas y seminarios/prácticas, y si, además, ii) los alumnos tomaran un papel de aprendizaje activo de los contenidos estructurantes mediante la realización de actividades en aula, basadas en material que el estudiante ha trabajado de forma no presencial.

Para llevar a cabo este proyecto hemos empleado la metodología de ciclos de mejora docenteCIMA, realizados en el Programa de Formación e Innovación Docente del Profesorado (FIDOP), pero la hemos combinado con el modelo de clase/aula inversa. La evaluación de la actividad, según la técnica de escaleras de aprendizaje y evaluación, opiniones de los alumnos, y autoevaluación, ofrece algunos puntos de mejora, pero sugiere que la intervención ha sido acertada en líneas generales.

Palabras clave: Medicina, Anatomía Patológica, docencia universitaria, experimentación docente universitaria, Clase inversa, Aprendizaje Basado en problemas

\section{Abstract}

Our hypothesis is that students' learning would improve if: i) the procedural contents were better integrated with conceptual ones throughout classroom improvement cycles (CIMA) that break the traditional dichotomy between theoretical and seminars/practical sessions, and if, in addition, ii) the students would take an active learning role of the structuring contents by carrying out classroom activities, based on material that the student has worked in a non-face-to-face manner.

To carry out this project we have used the methodology of learning improvement cycles (CIMA), carried out in the Training and Innovation Program for Teachers (FIDOP), but we have combined it with the inverse/flipped class/learning model. The evaluation of the activity, according to the technique of learning and evaluation ladders, student opinions, and self-evaluation, offers some points of improvement, but suggests that the intervention has been successful in general.

Keywords: Medicine, Pathology, University Teaching, University Teaching Experimentation, Flipped Class, Problem Based Learning. 


\section{Contexto de la asignatura}

La asignatura donde estoy realizando los ciclos de mejora es "Anatomía Patológica", correspondiente al primer semestre del tercer curso del grado de Medicina. Consta de 9 créditos repartidos, cada semana, en cuatro clases teóricas más 3 horas de prácticas o seminarios de una hora. Es una asignatura troncal y de formación básica (obligatoria), que se imparte en el primer cuatrimestre del curso. Los alumnos ya han cursado Histología y Anatomía. Consta de 50 temas, agrupados en 8 secciones temáticas. En el curso 2019-2020, no imparto solamente la docencia teórica sino también las prácticas y los seminarios. Esto me ofrece una oportunidad única para mejorar el aprendizaje de los alumnos integrando la formación teórica con la práctica centrándola más en los contenidos procedimentales clínicos.

Conexión con el diseño previo:Las principales conclusiones que se desprenden del trabajo del curso pasado son:

Avances conseguidos: Los alumnos se están acostumbrando y disfrutan de la docencia centrada en casos clínicos. Hay que tener en cuenta que son alumnos de tercero en un Grado de 6 años, y que es la primera asignatura abiertamente médica que reciben.

Dificultades encontradas:

- El modelo metodológico aplicado en el curso 20182019 en el que la explicación de casos prácticos compite en el tiempo (dentro de la misma clase teórica) con la explicación de los contenidos conceptuales correspondientes a dichos casos prácticos ha sido un inconveniente para los alumnos al principio del semestre porque aún no se sienten suficientemente seguros en el razonamiento clínico. La posibilidad de dedicar a la misma área temática dos formatos 
diferentes de docencia (clase teórica y seminario, por ejemplo) en dos días correlativos creo que puede ser una oportunidad para mejorar este aspecto, repartiendo e integrando los contenidos entre dos tipos de formatos presenciales de docencia.

- El nivel de participación en clase de los alumnos no es malo, pero es bastante desigual: se centra habitualmente en un $20 \%$ de los alumnos más participativos, el resto toma un papel más pasivo. Hace falta motivar involucrar a los alumnos desde la misma preparación de las clases. ¡El número de cuestionarios anónimos y voluntarios pre/post CIMA rellenados por los alumnos no llega al 10\% de los alumnos de cada grupo!

- El CIMA no3 se diseñó en el curso 2018-2019 para innovar la docencia de la segunda mitad del programa de la asignatura, dedicado a enfermedades concretas, y no a mecanismos generales de la enfermedad como los CIMA no1 y 2. Parece oportuno extender este tipo de docencia, muy centrada en casos clínicos, de manera más extensa a la segunda mitad del programa de la asignatura.

\section{Diseño previo de los CIMA:}

Se han diseñado 9 CIMA, que a partir de ahora denominaremos ciclo 1-9, y en los que se sincronizan e integran las clases teóricas con los seminarios y prácticas relacionados temáticamente.

1. El ciclo nô1 corresponde al área temática de bases cardiovasculares de la enfermedad. Corresponde a los temas 7-9 del temario. Fue objeto del primer CIMA del Curso Básico, pero ahora se consolida y completa con un sistema de evaluación completo, con el Seminario nô1 y la práctica no1.

Jornadas de Formación e Innovación Docente del Profesorado | № 2 (2019) Esta obra se distribuye con la licencia Creative Commons Reconocimiento-NoComercial-SinObraDerivada 
2. El ciclo no 2 corresponde al área temática de las neoplasias, temas 17-21 del temario. Se mantiene como CIMA integrado con la asignatura de Histología en Primer Curso del Grado de Medicina. En este caso se completa con el seminario no4 y la práctica no2.

3. En la segunda mitad de la asignatura se añaden 6 CIMAs al CIMAno3. Es un intento de innovar la docencia de la segunda parte del programa, la dedicada a las características concretas de cada enfermedad. En este caso los contenidos dejan de ser conceptuales para ser, en su mayor parte procedimentales o datos. He elegido para ello siete secciones del programa, apoyando en todos los casos las clases teóricas en clases prácticas o seminarios previos con casos clínicos sencillos y adaptados a los estudiantes de tercer curso de Medicina. Se adjunta una tabla con el resumen de la actuación:

Tabla 1. Características de cada CIMA propuesto:

\begin{tabular}{|c|c|c|c|c|c|}
\hline CIMA ne & Nombre del CIMA & $\begin{array}{l}\text { temas de teoría } \\
\text { integrados en el CIM, }\end{array}$ & $\begin{array}{l}\text { seminarios integrados } \\
\text { en el CIMA }\end{array}$ & $\begin{array}{l}\text { prácticas integradas en } \\
\text { el CIMA }\end{array}$ & $\begin{array}{l}\text { Total de horas } \\
\text { presenciales }\end{array}$ \\
\hline 1 & Patología vascular & nะ 7-9 & no1 & no1 & 5 \\
\hline 2 & $\begin{array}{l}\text { Patología general } \\
\text { de las neoplasias }\end{array}$ & no 17-21 & n4 & n으 & 7 \\
\hline 3 & $\begin{array}{c}\text { Carcinoma de } \\
\text { pulmón }\end{array}$ & no 30 & nะ5 & & 2 \\
\hline 4 & $\begin{array}{c}\text { Neoplasias de } \\
\text { intestino grueso e } \\
\text { hígado }\end{array}$ & nะ 32-36 & $n \div 6$ & n?3 & 7 \\
\hline 5 & $\begin{array}{l}\text { Neoplasias de } \\
\text { próstata }\end{array}$ & n38 & n7 & & 2 \\
\hline 6 & $\begin{array}{c}\text { Neoplasias de la } \\
\text { mama }\end{array}$ & $n \div 44$ & $n \div 8$ & nะ4 & 2 \\
\hline 7 & $\begin{array}{c}\text { Neoplasias del } \\
\text { tiroides }\end{array}$ & n-45 & & n5 & 2 \\
\hline 8 & $\begin{array}{l}\text { Neoplasias del } \\
\text { sistema nervioso } \\
\text { central }\end{array}$ & no48 & n9 & & 2 \\
\hline 9 & $\begin{array}{c}\text { Neoplasias } \\
\text { melanocíticas }\end{array}$ & n?50 & n¹0 & & 2 \\
\hline Total horas & & & & & 31 \\
\hline
\end{tabular}

Jornadas de Formación e Innovación Docente del Profesorado | № 2 (2019) Esta obra se distribuye con la licencia Creative Commons Reconocimiento-NoComercial-SinObraDerivada Internacional (CC BY-NC-ND 4.0.) 


\section{Principios didácticos argumentados comunes a los 9 ciclos}

Respecto a los contenidos he hecho una reflexión sobre los contenidos más estructuradores (p.ej. concepto de neoplasia, cómo diagnosticar una neoplasia), los más conceptuales y los procedimentales. Además, se han incluido habilidades procedimentales como la de describir lesiones o la de hablar en público. También se han incluido valores, como el de la prevención o la investigación.

Respecto al modelo metodológico, éste se ha basado en el trabajo de casos clínicos de forma individual o grupal de manera previa o durante la clase, de forma que ayude a los estudiantes a ser capaces de relacionar los conceptos teóricos con la realidad clínica. Parece importante tener una interacción muy estrecha con los alumnos durante la preparación de las clases, invitándoles a aprovechar este momento para exponerse a la materia. Para el profesor, este tiempo es privilegiado para conocer de manera específica (mediante cuestionarios especialmente diseñados a tal efecto) qué es lo que les cuesta más comprender a los alumnos y para introducir cambios que favorezcan la mejora del aprendizaje. Estos principios se han basado en las recomendaciones de Bain (2007) y Finkel (2005) sobre el aprendizaje autónomo de los alumnos. También se han incluido algunos elementos de aprendizaje inverso o inductivo "flipped learning", según las recomendaciones de Prieto (2018).

\section{Modelo metodológico argumentado común para los 9 ciclos}

En el modelo metodológico seguido en los CIMA, Comenzamos el estudio de cada bloque temático con el debate de casos clínicos (en formato seminario o práctica), y se continúa con las explicaciones teóricas que ayudan a 
la resolución de estos, (en formato clase teórica) de la siguiente forma:

- Como trabajo previo a la sesión, el profesor sube a EV los materiales propuestos en relación con el caso clínico (CP) que van a servir de hilo conductor para comprender los contenidos teóricos

- Además, de manera previa a la clase el profesor envía cuestionarios de comprobación de estudio en Google Forms que sirven para verificar el trabajo del alumno antes de clase, para hacer llegar feedback al profesor acerca de las dudas más urgentes y de las preguntas más interesantes, lo que permite al profesor replantear los contenidos de la clase de manera previa a su impartición, y para ayudar a construir las escaleras de aprendizaje.

- Se inicia la práctica o el seminario con la exposición del caso clínico, y con un trabajo de debate de este en forma de preguntas intercaladas con discusión por parejas o por pequeños grupos y explicaciones aclaratorias. La práctica o seminario termina con cuestionarios de comprobación de aprendizaje mediante sistemas de gamificación tipo Kahoot dirigidos a centrar a los alumnos sobre algún tema clave de la sesión y a saber qué conceptos previos han conocido; las respuestas se envían de manera anónima y se programa el aplicativo para que no salga la respuesta correcta, de tal manera que se mantiene el 'suspense' y con ello la atención.

- Durante el tiempo no presencial que sigue, los alumnos pueden dirigir dudas y preguntas al profesor mediante un cuestionario específico, y éste está a tiempo de reformular la clase en lo que sea necesario.

- En la siguiente clase teórica, se comienza dando feedback a los alumnos acerca de las dudas más relevantes o urgentes. Posteriormente hay una breve explicación teórica de 30 minutos (T). A continuación, se repite el televoto mediante sistemas de gamificación tipo Kahoot (EV); se trata de las mismas preguntas que al principio de la clase; en este caso el sistema 
pregunta su nombre a cada alumno y le proporciona la respuesta correcta (2 minutos). El profesor puede así comprobar in situ si ha habido o no aprendizaje de los cometidos deseados durante la sesión. La sesión acaba con un feedback final por parte del profesor.

- En el tiempo que sigue a la clase, los alumnos vuelven a tener acceso al mismo cuestionario de comprobación de estudio en Google Forms, lo que permite al profesor evaluar el CIMA y construir las escaleras de aprendizaje. Todo ello queda recogido en la Figura 1.

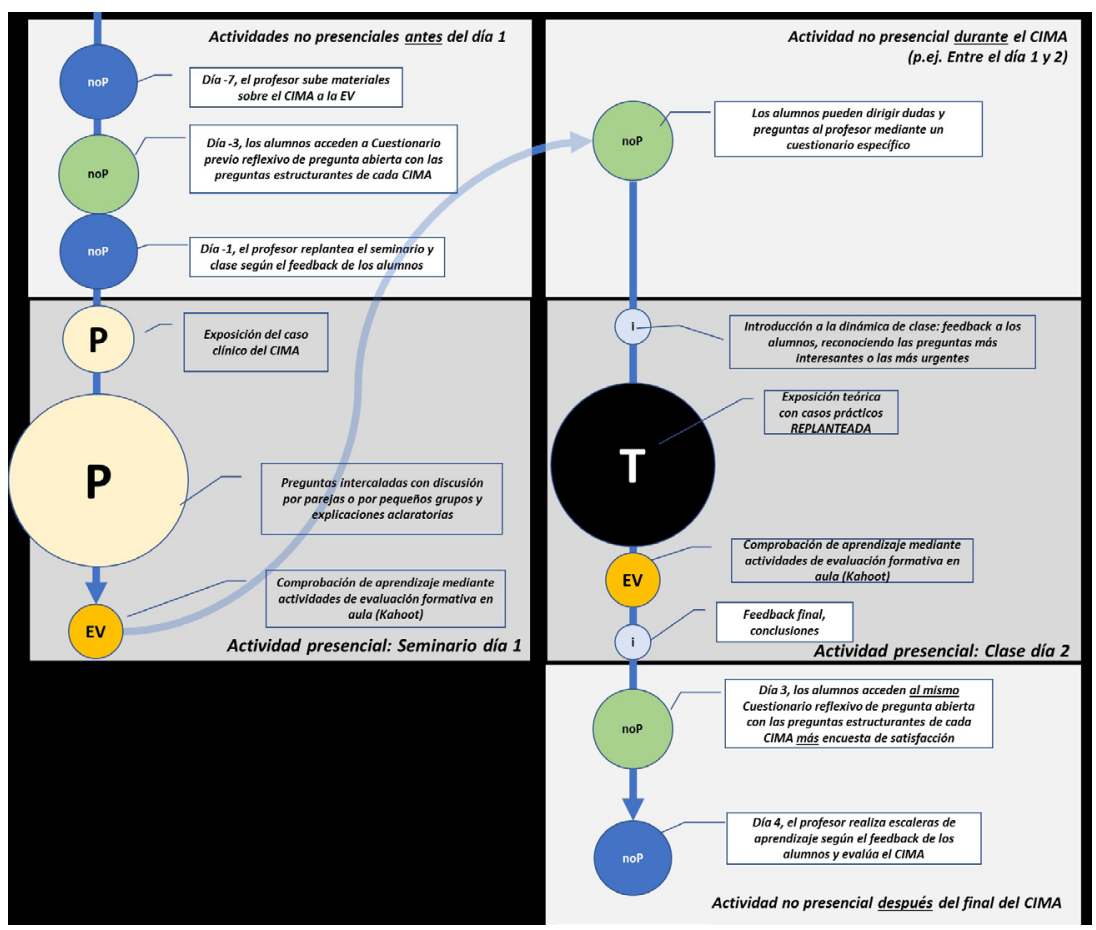

Figura 1: Modelo metodológico común a los 9 CIMA

Leyenda: P: Contenido práctico en relación con el caso clínico. noP: Actividad personal del alumno del profesor. I: introducción a la dinámica de clase. EV: Evaluación mediante televoto. T: Exposición teórica por parte del profesor de los contenidos teóricos en los que se apoya la resolución de las preguntas planteadas en el caso clínico.

A continuación, detallo los aspectos específicos de cada ciclo de mejora: 


\section{Primer CIMA}

\section{Mapa de contenidos y problemas o preguntas clave}

Este primer ciclo de mejora se lleva a cabo en cuatro sesiones de 55 minutos en los que se imparten los temas referentes a las bases de la enfermedad relacionadas con la circulación de la sangre. Son temas importantes, aunque relativamente fáciles, y hacen por ello que la introducción del nuevo modelo metodológico por primera vez en el Grado no desconcierte, sino que estimule a los estudiantes.

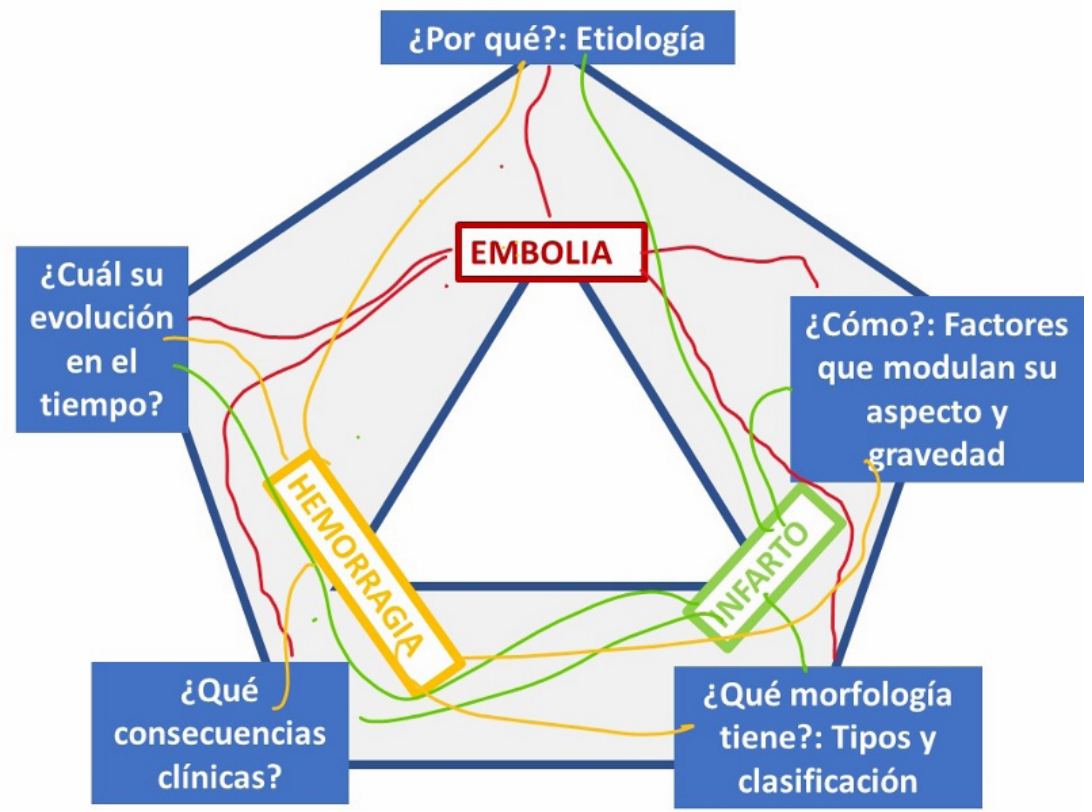

Figura 2. Mapa de contenidos de la sección de mecanismos de enfermedad circulatoria. El gráfico representa las cinco preguntas clave de las tres lesiones básicas de la enfermedad circulatoria (hemorragia, embolia, infarto). Las cinco preguntas están relacionadas entre si (líneas gruesas), lo mismo que las tres lesiones clave. Las líneas finas de color representan las relaciones entre cada uno de estos contenidos.

Jornadas de Formación e Innovación Docente del Profesorado | № 2 (2019) Esta obra se distribuye con la licencia Creative Commons Reconocimiento-NoComercial-SinObraDerivada 


\section{Evaluación:}

Se realizó una evaluación del estudiante durante el proceso de aprendizaje mediante cuestionarios, cuyas respuestas se analizaron según el modelo de escaleras de aprendizaje propuesto por FIDOP. Se incluyó también una evaluación del estudiante tras el proceso de aprendizaje. Por último, existe un sistema de evaluación del docente y del diseño didáctico mediante una estructura de autoevaluación y de evaluación del diseño de los ciclos de mejora.

A continuación, se señalan los enlaces a:

- el cuestionario que se dirigió a los alumnos ANTES del CIMA nㅇ: https://forms.gle/eEVqs8YfXs44KHwX8

- el cuestionario que se mantuvo abierto DURANTE el CIMA nㅇ: https://forms.gle/JCbRHhZd4hBmBMHf9

- y el cuestionario que se dirigió a los estudiantes DESPUÉS del CIMA no1, similar al previo, pero con preguntas específicas para que evaluaran toda la experiencia, incluidos los materiales docentes: https:// forms.gle/3ZsoXNaXxfCyVKr68

Se emplearon cuestionarios análogos a los anteriores para cada uno de los CIMAs

\section{Segundo CIMA}

\section{Mapa de contenidos y problemas o preguntas-clave}

El ciclo de mejora se realiza en seis sesiones de $55 \mathrm{mi}-$ nutos de clase, en las cuales se imparten los temas referentes a las neoplasias. Las neoplasias son un tema central en la Anatomía Patológica, y tanto los alumnos como el profesor están concienciados de su importancia dentro del temario. A continuación, muestro, por un lado, el mapa de contenidos general del CIMA, y, a continuación, el mapa de la actuación integrada con Histología. 
El mapa de contenidos general del CIMA planteado es similar al de años previos y se representa en la Figura 3

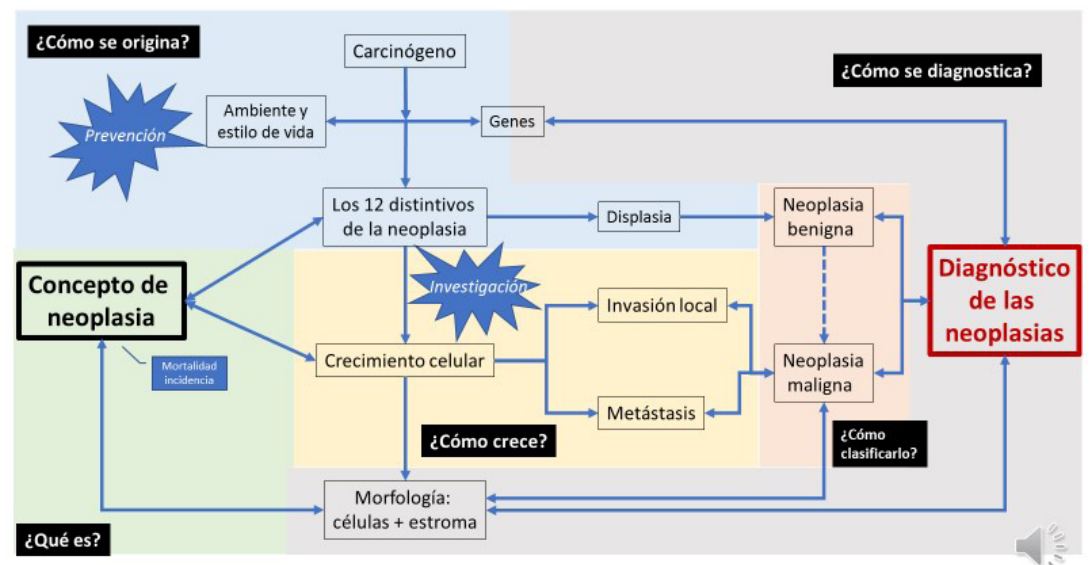

Figura 3: Mapa de contenidos general del segundo CIMA

Leyenda: Conceptos fundamentales: negrita. Contenidos conceptuales: Negro; contenidos procedimentales: rojo. Estrellas: valores. Flechas: relaciones entre contenidos. Flecha discontinua: relación probable.

A continuación, se detallan las preguntas clave:

1. ¿Qué es o cómo se define una neoplasia?

1.bis ¿Cuáles son las características más importantes en la estructura normal del estroma conjuntivo de las neoplasias? ¿Qué aspectos de la estructura y la función del estroma influyen en la respuesta clínica del cáncer?

2. ¿Cómo se origina una neoplasia?

3. ¿Cómo crece y se disemina una neoplasia?

4. ¿Cómo se clasifica o se nombra una neoplasia?

5. ¿Cómo se diagnostica una neoplasia?

Se incluye un enlace a un vídeo cargado en la plataforma Youtube, donde describo la construcción del mapa de contenidos y cómo se integran las preguntas clave en el esquema: https://youtu.be/4XpwC A-GWY

La pregunta clave no1bis, al ser la pregunta que define la parte integrada con Histología, tiene un mapa de 
contenidos independiente pero que debería verse como un detalle integrado en el mapa mostrado con anterioridad (concretamente dentro del contenido "Morfología: células+estroma" de la pregunta clave "Diagnóstico de la neoplasia")

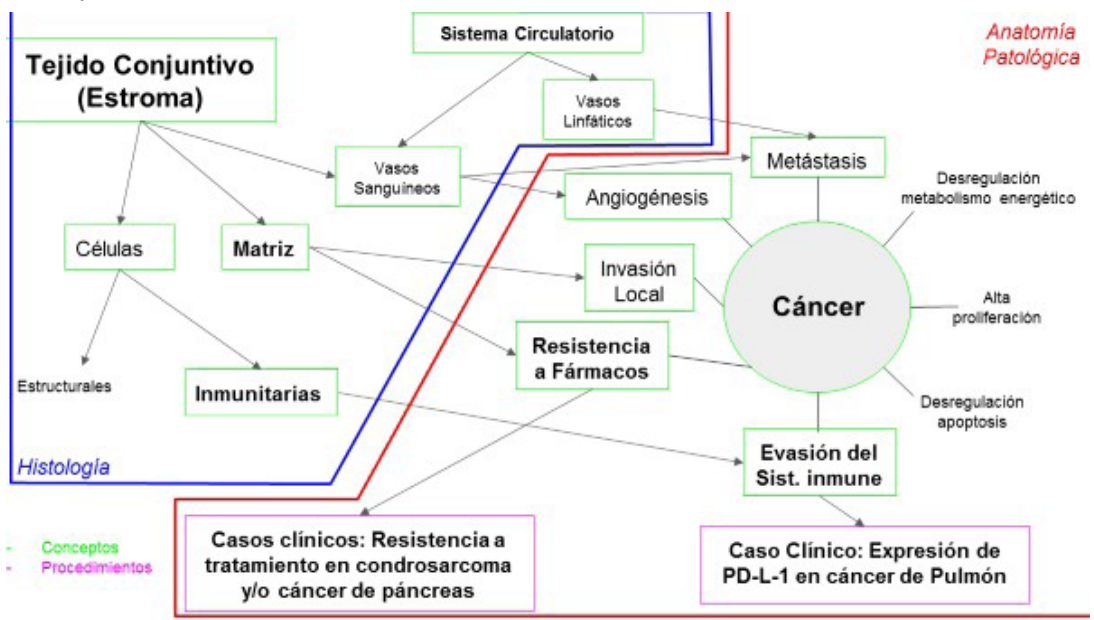

Figura 4. Mapa de contenidos de la parte integrada con Histología del CIMA no2.

\section{CIMAs 3-9}

\section{Mapa de contenidos y problemas o preguntas clave}

Estos 7 CIMAs se llevan a cabo en entre 2 y 7 sesiones de 55 minutos, combinando clases teóricas, prácticas y seminarios, como se establece en la Tabla no1. Estos CIMAs están situado en la parte final del programa. Esto ayuda a los alumnos (iy al profe!) a no relajarse tras los dos primeros ciclos de mejora. Además, los contenidos cambian respecto a la primera parte del programa, pues ya no se trata tanto de comprender los mecanismos básicos de la enfermedad, sino que describimos ahora enfermedades concretas. Es posible que en el curso 2020-2021 alguno de estos CIMA pueda convertirse en CIMA integrados con Patología Médica y Quirúrgica de 4으 o 5으 curso de Medicina. 


\section{ENRIQUE dE ÁLAVA CASADO}

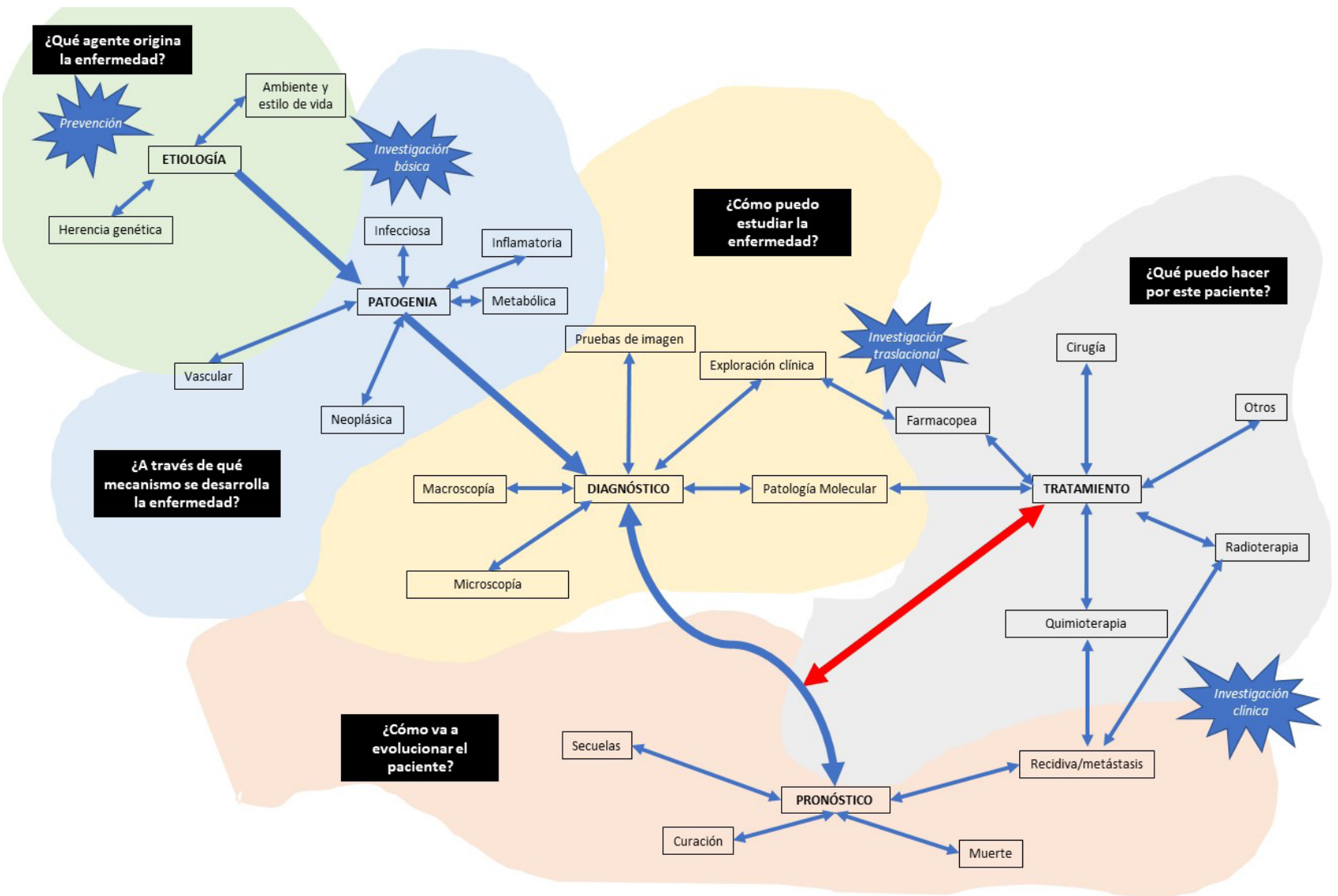

Jornadas de Formación e Innovación Docente del Profesorado I № 2 (2019)

cC (i) $\Theta$ Esta obra se distribuye con la licencia Creative Commons

cc) $(1) \Theta$ Reconocimiento-NoComercial-SinObraDerivada

Internacional (CC BY-NC-ND 4.0.) 
Figura 5. Mapa de contenidos común a los CIMAs 3-9. Se observan las cinco preguntas estructurantes en cajas negras, centrando espacios de colores diferenciados donde se encuentran los contenidos y sus relaciones. Para un alumno de tercer curso de medicina todos los contenidos con cajas blancas son conceptuales, excepto los del área amarilla (diagnóstico), que son procedimentales. Algunos contenidos conceptuales se escriben con letras mayúsculas, dada su importancia. Se indican dos contenidos-valores con cajas de forma estrellada. Las flechas indican las relaciones entre los contenidos; su grosor y su color están en relación con su importancia

A continuación, se detallan las preguntas clave genéricas, para cada CIMA 'la enfermedad' se sustituye por el área temática de cada CIMA, por ejemplo, en el CIMA no3 'carcinoma de pulmón':

1. ¿Qué agente causa la enfermedad?

2. ¿A través de qué mecanismo se desarrolla la enfermedad?

3. ¿Cómo puedo estudiar la enfermedad?

4. ¿Cómo va a evolucionar el paciente?

5. ¿Qué puedo hacer por este paciente?

\section{Evaluación de los CIMA 3-9}

De manera análoga a los CIMA 1 y 2, con cuestionarios similares y escaleras de aprendizaje.

\section{Aplicación del CIMA}

En el momento de enviar esta comunicación, se han terminado y evaluado los tres primeros CIMAs, el cuarto está recién terminado y faltan por realizarse los CIMA 5-9

\section{CIMA n이}

\section{Resumen del diario de clase:}

El primer día (1 de octubre) empezamos el ciclo, tal y como habíamos quedado, con un seminario dedicado 
a una historia clínica de arteriosclerosis. Es la primera vez que ven un caso clínico en una clase en su vida, y les pongo una historia sencilla. Confieso que estoy un poco nervioso para ver cómo van a reaccionar, pero los veo muy interesados; les muestro las conexiones entre los contenidos procedimentales del seminario y los más conceptuales de las clases que vamos a ir teniendo en los próximos días. Me alegro de haber comenzado el CIMA con un caso clínico. Les doy feedback sobre el cuestionario previo al CIMA, que han respondido 91 personas (son 100 en clase). Lo acogen bien.

El día 2 de octubre imparto el tema teórico que estaba previsto. Un tema corto y fácil; todos disfrutamos de los ejemplos clínicos. Les aviso que ese día abro el cuestionario interno del ciclo. Esa misma noche activo el cuestionario interno del ciclo para que me hagan comentarios y preguntas.

El 3 de octubre impartimos el tema de la trombosis y la embolia. Noto que se quedan muy extrañados con el mecanismo de la embolia paradójica y no es para menos porque es una cosa compleja de entender. Esa misma noche aparecen preguntas en el cuestionario interno, incluyendo la necesidad de que expliqué más lo que es una embolia paradójica, así como qué es la coagulación intravascular diseminada. Preparo algunos contenidos relevantes y los subo a la enseñanza virtual.

El 4 de octubre abordamos el tema del infarto, y la clase, la adapto completamente a las preguntas que han hecho en las noches anteriores a través del cuestionario. Noto que tenemos buena conexión.

Al valorar los cuestionarios posteriores al CIMA no1 (que responden 88 personas) noto que tienen mucha inquietud por aprender más, y también una marcada satisfacción por el ciclo recién terminado (nota media 8,3/10). 


\section{Evaluación mediante escalera de aprendizaje}

Los cuestionarios de contenidos tenían 5 preguntas. He construido escaleras correspondientes a las 5 preguntas según la metodología descrita en las referencias bibliográficas de esta comunicación (Porlán R, 2017). Sin embargo, por razones de espacio, voy a mostrar la que considero más representativa por el notable progreso en el aprendizaje.

CIMA 1, pregunta 3. ¿Qué morfología pueden tener las lesiones derivadas de las tres alteraciones hemodinámicas?

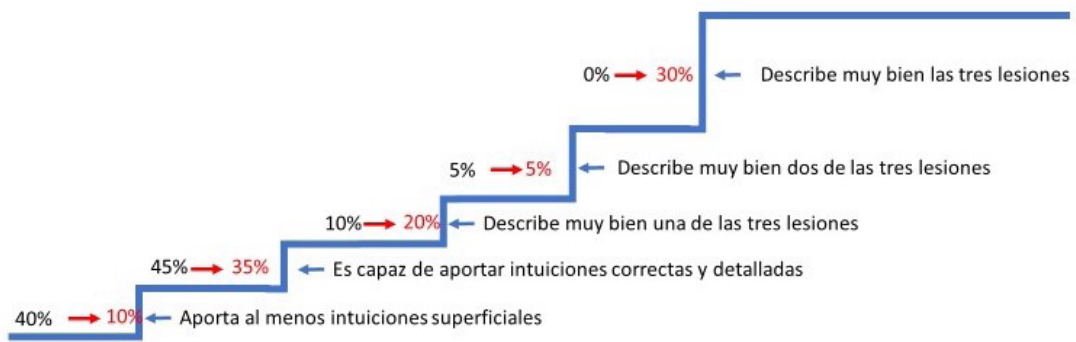

Figura 6. Escalera de aprendizaje de la pregunta no3 del CIMA nำ1

En la respuesta a esta pregunta se observa un adecuado avance en el aprendizaje de cuáles son las lesiones asociadas a la trombosis, hemorragia y embolia. Aunque muchos alumnos tenían intuiciones más o menos precisas acerca de dichas lesiones, aproximadamente el 35\% de ellos fueron capaces al final del ciclo de realizar una descripción satisfactoria de las lesiones. Como conclusión para la pregunta guía 3 del CIMA no1, creo que hay que insistir en la metodología docente ligada a este aspecto para el próximo curso.

Jornadas de Formación e Innovación Docente del Profesorado | № 2 (2019) Esta obra se distribuye con la licencia Creative Commons Reconocimiento-NoComercial-SinObraDerivada 


\section{CIMA n은}

\section{Resumen del diario de clase}

Comienzo el ciclo el 21 de octubre haciéndoles feedback acerca de lo que habían escrito en el cuestionario previo al CIMA no2 (87 personas han escrito el cuestionario previo). En particular en lo que tiene que ver con la definición de neoplasia. Para ello les construyo y subo a EV una nube de letras formada a partir de sus respuestas a la pregunta no 1 “QQué es una neoplasia?”. Esta no era una actividad prevista, pero se me ocurrió unos días antes y creo que sirvió muy bien para que vieran cuáles eran sus concepciones previas al CIMA. Nos quedamos casi toda la clase con este aspecto. Este año conservamos el material del CIMA integrado con Histología (Prof. Fernández Santos) pero no vi práctico que se desplazase a nuestra aula. Les dejo un pequeño problema/mini caso clínico relacionado con la segunda clase. Abro el cuestionario interno del ciclo para que me vayan dando feedback entre clase y clase.

El 22 de octubre comenzamos abordando el mini-caso clínico propuesto al final de la clase anterior, y nos quedamos un rato largo debatiéndolo. Los contenidos de la clase, relacionados con la diseminación de las neoplasias son extensos, y eso que los he recortado respecto al curso anterior (79 diapositivas respecto a 140), pero aun así dejo algunas para el comienzo de la clase siguiente. Muy buen clima de participación en clase, pero pocas preguntas en el cuestionario.

El 23 de octubre lo dedico a un seminario muy práctico sobre nomenclatura tumoral, donde preparamos entre todos una clasificación de las neoplasias 'for dummies'. Contra lo que tenía previsto, me centro en que comprendan 
que la palabra pólipo es muy ambigua, y que no necesariamente refleja una naturaleza neoplásica. No termino todos los contenidos del seminario, pero doy por buena la experiencia porque a partir de ahora queda muy clara la nomenclatura de las neoplasias.

El 24 de octubre acabo los contenidos de la clase anterior y abordo un mini caso clínico para centrar el tema de la etiología del cáncer. Todo según lo previsto. Pocas preguntas en el cuestionario.

El 25 de octubre revisamos en clase las bases genéticas de las neoplasias. Noto que les cuesta entender el modelo de Knudson relacionado con el retinoblastoma y los genes supresores tumorales, y me propongo inventar algo al respecto para el próximo curso. Subo a EV un artículo que se publicó en Nature el día anterior sobre las alteraciones genéticas de las metástasis, pero me doy cuenta de que es demasiado complejo para casi todos ellos.

Termino el ciclo el 28 de octubre, cerrando todo el CIMA alrededor del diagnóstico de las neoplasias. Es una clase dinámica en la que todos disfrutamos.

Al acabar el ciclo subo a EV mi vídeo con el mapa de contenidos (Figura 3 de este artículo) y los alumnos me reflejan que les ayuda a aprender. Los cuestionarios posteriores al CIMA n은 los responden 78 personas.

\section{Evaluación mediante escalera de aprendizaje}

Los cuestionarios de contenidos tenían 5 preguntas. He construido escaleras correspondientes a las 5 preguntas según la metodología descrita en las referencias bibliográficas de esta comunicación (Porlán R, 2017). Sin embargo, por razones de espacio, voy a mostrar la que considero más representativa por la falta de progreso en el aprendizaje 
CIMA 2, pregunta 2. ¿Cómo se diseminan las neoplasias?

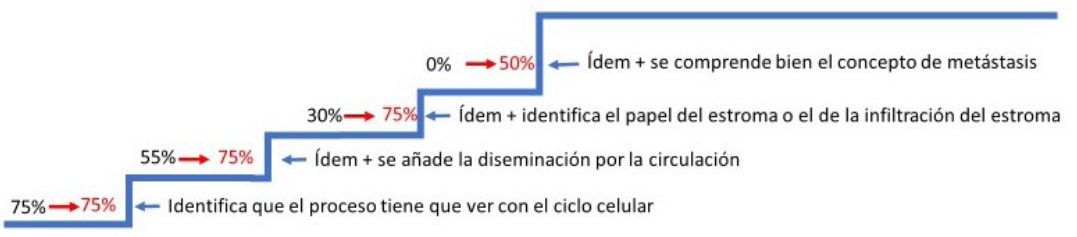

Figura 7. Escalera de aprendizaje de la pregunta no2 del CIMA no2

En particular, me llama la atención de que el concepto de metástasis no se haya captado adecuadamente, pues solo el $50 \%$ de los cuestionarios evaluados muestra una comprensión adecuada de este contenido, aunque ciertamente el progreso es notable al partir desde un 0\%. Continuaremos insistiendo de manera específica en el aprendizaje de este contenido.

\section{CIMA n으}

\section{Resumen del diario de clase:}

Este es un CIMA de solo 2 sesiones centrado en el carcinoma de pulmón. Al ser un CIMA nuevo me requiere mucho trabajo previo de adaptación de materiales previos. Asocio a la docencia de este CIMA a un MIR de Anatomía Patológica que prepara e imparte conmigo una clase teórica. El cuestionario previo lo responden 80 personas, y no subo materiales previos. Sin embargo, entre la primera y segunda sesión, en lugar de subir un cuestionario interno, subo dos vídeos de YouTube sobre el diagnóstico y el tratamiento de los carcinomas de pulmón, que son muy bien evaluados por los alumnos, y que comentamos en la segunda clase. 
Comenzamos las actividades presenciales del CIMA no3 el 13 de noviembre con un seminario en el que tienen ocasión de ver 6 casos diferentes de neoplasias pulmonares; cada uno de los casos acaba con un diagnóstico diferencial y un kahoot para conocer qué diagnóstico creen los estudiantes que es más probable. El seminario sirve para que los estudiantes identifiquen algunos contenidos clave (diferenciación neuroendocrina, expresión de TTF-1, localización intrapulmonar, etc.) en los que se profundizó en clase teórica.

El 14 de noviembre tuvo lugar la clase teórica del CIMA. La impartí junto con el Dr. Fernando Zapata, MIR de Anatomía Patológica. Vi que los alumnos seguían muy bien sus explicaciones, y de hecho agradecieron que hubiera una voz nueva. Para él fue su primera clase $y$, aunque le di varios consejos de mejora, creo que fue una muy buena clase. Los alumnos participaron quizás un poco menos de los habitual.

El cuestionario posterior al CIMA lo respondieron 88 personas (se nota que ya ha pasado el parcial de microbiología). El índice de satisfacción fue de 8,3/10.

\section{Evaluación mediante escalera de aprendizaje}

Los cuestionarios de contenidos tenían 5 preguntas. He construido escaleras correspondientes a las 5 preguntas según la metodología descrita en las referencias bibliográficas de esta comunicación (Porlán R, 2017). Sin embargo, por razones de espacio, voy a mostrar la que considero más representativa por la falta de progreso en el aprendizaje. 
CIMA 3, pregunta 3. ¿Cómo puedo estudiar el carcinoma pulmonar?

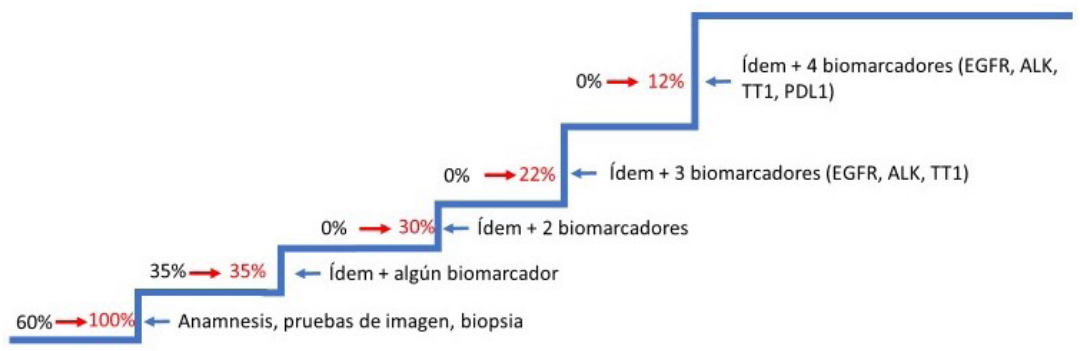

Figura 8. Escalera de aprendizaje de la pregunta no3 del CIMA no3

La razón fundamental por la que selecciono esta pregunta es porque, aunque los alumnos demuestran tener el conocimiento básico de las herramientas diagnósticas, no han sabido identificar los biomarcadores necesarios para la detección de dianas terapéuticas en el carcinoma pulmonar (EGFR, ALK, etc.). Por lo tanto, habrá que insistir en ello en los próximos cursos

\section{Evaluación de los CIMA. Conclusiones}

Avances conseguidos:

- Hemos sido capaces de centrar la docencia en casos (problemas clínicos reales) y de hecho hemos empezado cada CIMA con casos prácticos. Para no crear inseguridad en los estudiantes, hemos incluido "clases teóricas' inmediatamente después, para consolidar los conocimientos aprendidos. Así, hemos dedicado a la misma área temática dos formatos diferentes de docencia (clase teórica y seminario, por ejemplo) en dos dias correlativos, repartiendo e integrando los contenidos entre los dos tipos de formatos presenciales de docencia. El resultado es que la diferencia entre clases teóricas y prácticas/seminarios se ha difuminado notablemente. 
- El nivel de participación de los estudiantes ha sido notablemente mejor, lo que se ha favorecido por haber asignado (ver siguiente apartado) un 25\% de la calificación a la cantidad y calidad de la participación en los cuestionarios. Si en el curso pasado la participación era de 5-8 alumnos por cuestionario, ésta no ha bajado de los 80 en el curso actual.

- De manera complementario al punto anterior, hemos introducido aspectos de clase invertida en nuestra docencia y hemos sido capaces de modular los contenidos de algunas clases teóricas gracias a los cuestionarios que, durante los CIMA 1 y 2, hemos abierto a la participación de los estudiantes.

- Estamos siendo capaces de extender el modelo de ClMAs a la segunda mitad del temario (CIMAs 4-9, aún en desarrollo).

\section{Aspectos a mejorar:}

- Existen algunas clases cuyos materiales aún son demasiado largos y complejos, y necesitarían ser jerarquizados y resumidos

- Convendría escribir pies de figura en algunas figuras con contenidos complejos.

- Las escaleras de aprendizaje revelan que hay algunos contenidos (p.ej. biomarcadores en el carcinoma de pulmón, concepto de metástasis) en los que se debe mejorar el aprendizaje, para lo que deberemos pensar nuevas estrategias docentes.

\section{Sistema de calificación del curso}

Para este curso está en vigor la siguiente tabla de calificaciones:

- Examen final: 70\%, centrado de manera mayoritaria en los casos debatidos en los CIMAs 
- Participación mediante cuestionarios (Google Forms y gamificación Kahoot). Se valora la frecuencia y la calidad de la participación: 25\%

- Realización de la encuesta oficial de la Universidad de Sevilla de opinión del alumnado (se verificará mediante un cuestionario específico en Google Forms, cuyo enlace es: https://forms.gle/z2z1uYcVFyD6ufTe6): 5\% 


\section{Bibliografía}

Blain, K. (2007). Lo que hacen los mejores profesores de Universidad. Valencia: Publicaciones de la Universitat de Valéncia.

Finkel, D. (2008). Dar clase con la boca cerrada. Valencia: Publicaciones de la Universitat de Valéncia.

Porlán R (Coord.) (2017). Enseñanza Universitaria, cómo mejorarla. Madrid. Ediciones Morata.

Prieto Martín A (2018). Flipped learning. Aplicar el modelo de aprendizaje inverso. Madrid. Ediciones Narcea.

Jornadas de Formación e Innovación Docente del Profesorado | № 2 (2019) Esta obra se distribuye con la licencia Creative Commons 